\title{
Mesocopic comparison of complex networks based on periodic orbits
}

\author{
Stoop, R ; Joller, J
}

\begin{abstract}
Complex noiseless dynamical systems can be represented in a compressed manner by unstable periodic orbits. It is unknown, however, how to use this technique to obtain a suitable notion of similarity among them, how to extend such an approach to more general complex networks, and how to apply such a method in the important case of noisy systems. Our approach provides a solution to these questions. For a proof-of-concept, we consider Drosophila's precopulatory courtship, where our method reveals the existence of a complex grammar (similar to those found in complex physical systems and in language), leading to the conclusion that the observed grammar is very unlikely the product of chance.
\end{abstract}

DOI: https://doi.org/10.1063/1.3553643

Posted at the Zurich Open Repository and Archive, University of Zurich ZORA URL: https://doi.org/10.5167/uzh-48727

Journal Article

Published Version

Originally published at:

Stoop, R; Joller, J (2011). Mesocopic comparison of complex networks based on periodic orbits. Chaos, 21(1):016112.

DOI: https://doi.org/10.1063/1.3553643 


\section{AIP $\left.\right|_{\text {chaos }}$ \\ An Interdisciplinary Joumal of Nonlinear Science}

\section{Mesocopic comparison of complex networks based on periodic orbits}

R. Stoop and J. Joller

Citation: Chaos 21, 016112 (2011); doi: 10.1063/1.3553643

View online: http://dx.doi.org/10.1063/1.3553643

View Table of Contents: http://scitation.aip.org/content/aip/journal/chaos/21/1?ver=pdfcov

Published by the AIP Publishing

\section{Articles you may be interested in}

Topology identification of uncertain nonlinearly coupled complex networks with delays based on anticipatory synchronization

Chaos 23, 013127 (2013); 10.1063/1.4793541

Nucleation pathways on complex networks

Chaos 23, 013112 (2013); 10.1063/1.4790832

Reverse engineering of complex dynamical networks in the presence of time-delayed interactions based on noisy time series

Chaos 22, 033131 (2012); 10.1063/1.4747708

Identifying complex periodic windows in continuous-time dynamical systems using recurrence-based methods

Chaos 20, 043130 (2010); 10.1063/1.3523304

Synchronization-based scalability of complex clustered networks

Chaos 18, 043109 (2008); 10.1063/1.3005782

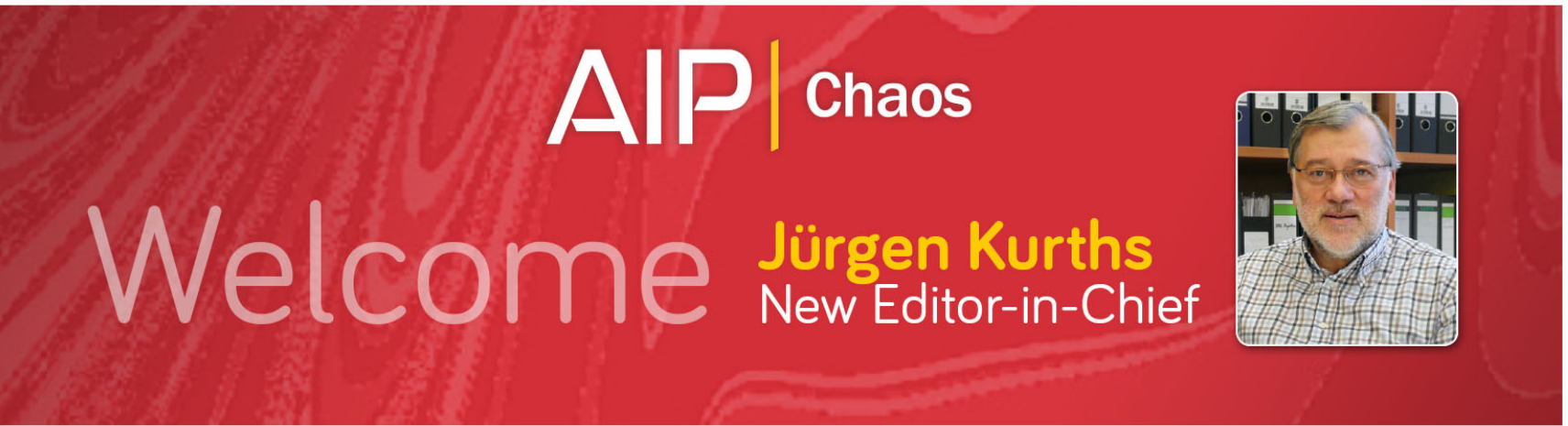




\title{
Mesocopic comparison of complex networks based on periodic orbits
}

\author{
R. Stoop ${ }^{1, a)}$ and J. Joller ${ }^{2}$ \\ ${ }^{1}$ Institute of Neuroinformatics, University of Zürich and ETH Zürich, Winterthurerstr. 190, 8057 Zürich, \\ Switzerland \\ ${ }^{2}$ University of Applied Sciences Rapperswil HSR, Oberseestrasse 10, 8640 Rapperswil, Switzerland
}

(Received 5 November 2010; accepted 19 January 2011; published online 29 March 2011)

\begin{abstract}
Complex noiseless dynamical systems can be represented in a compressed manner by unstable periodic orbits. It is unknown, however, how to use this technique to obtain a suitable notion of similarity among them, how to extend such an approach to more general complex networks, and how to apply such a method in the important case of noisy systems. Our approach provides a solution to these questions. For a proof-of-concept, we consider Drosophila's precopulatory courtship, where our method reveals the existence of a complex grammar (similar to those found in complex physical systems and in language), leading to the conclusion that the observed grammar is very unlikely the product of chance. (C) 2011 American Institute of Physics. [doi:10.1063/1.3553643]
\end{abstract}

Complex networks are usually described in terms of abstract network structures, where a node often comes equipped with a rule characterizing its local activity, or by using Markov random walks models. For their understanding, however, intermediate scales may play a distinguished role. This is the case if measures characterizing the network as a whole are too crude for providing the desired insights and if the local node dynamics do not provide an overview of the network. We formulate here in precise mathematical terms such as intermediate, mesoscopic, description of the network, based on periodic orbits as the mesoscopic observables. From these observables, we develop a method for measuring the similarity of graphs, a challenge of great importance that seems not to have been solved in the generality put forward here. An obvious typical application of our approach would be, e.g., the characterization of behavior, with robotics motion planning or the characterization of animal courtship behavior as prominent examples. Animal courtship, which we chose as the real-world testing case of our approach, is a puzzling phenomenon. Its function and purpose have largely remained unexplained, not least because of the generally large and complex underlying network structure and the lack of methods able to deal with such situations. The driving biological questions that we wish to answer here are: Could it be that during courtship, nontrivial information about a prospective mate is conveyed? And if so, how is this information organized and what purpose could it serve? The proposed approach provides partial answers to these questions. We verify that Drosophila courtship is heavily organized along periodic orbits, much more than would be expected from surrogate data. This indicates that the courtship is governed by a highly variable and complex language grammar, distinct from a simple random walk on the underlying graph. Using this grammar the individual can identify itself as a member of a genetic or developmental class, e.g., as a mature male. Moreover,

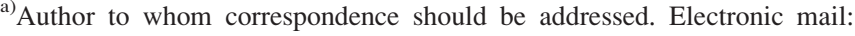
ruedi@ini.phys.ethz.ch. and probably even more importantly, it could also be used for the encoding and transmission of an individual's personalized footprint to the courtship partner. Such ability could play a decisive role in evolutionary optimization.

\section{INTRODUCTION}

Consider a complex directed graph in a theoretical or an experimental setting, such as the graph of Drosophila's precopulatory courtship $(D P C)$ behavior of Fig. 1. How can we distinguish this particular network from other networks? How can we formulate a fine-tuned notion of "similarity" between such networks and how could one assert that one network is more complex than another? To address these questions, an intermediate scale network analysis is needed. In this contribution, we develop a general method that characterizes complex networks on such a mesoscopic level. We demonstrate that by using this approach, questions of the above type become tractable.

The basic concepts for such a mesoscopic analysis already exist. Irreducible unstable periodic orbits (IUPO) have been shown to capture the complexity of chaotic systems in a condensed manner. ${ }^{1-7}$ As IUPO are generally composed of a number of network elements, and since the whole network can be exhausted by IUPO in an efficient and well-controlled manner, they are highly suitable for a mesoscopic description of complex networks. In the past, IUPO have been used to extract salient, essentially macroscopic, properties from dynamical systems. ${ }^{1-7}$ We will show that a similar approach allows one to extract mesoscopic properties from general complex networks, where the dynamical notion of "instability" is generally replaced by a corresponding probabilistic branching property. We will finally demonstrate how, upon a specification of the general method, we can cope with the presence of noise. This feature not only is decisive for the successful treatment of our proof-of-concept application, it more generally demonstrates that with this specification the method is applicable to an important class of real-world situations. 


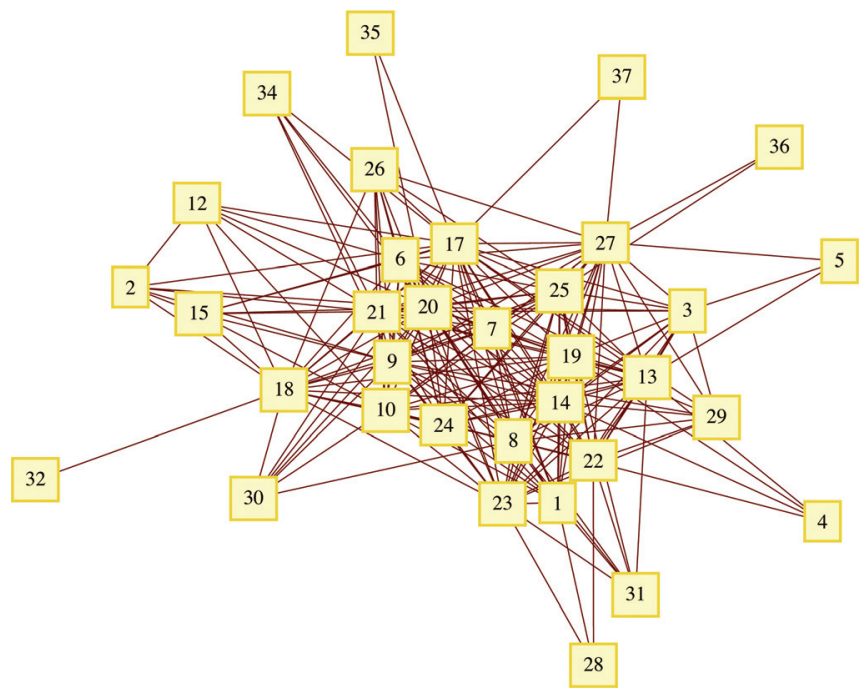

FIG. 1. (Color online) Undirected behavioral $D$ courtship graph, from male (normal and fruitless) and female (in different developmental stages), based on five individuals each. In $D$ courtship, we identify 37 fundamental courtship elements.

\section{GRAPH FUNDAMENTALS}

Let us first outline the general mathematical framework of graphs, orbits, and cycles. Let $G$ be a directed graph consisting of a set of vertices/nodes $V(G)$ and a set of edges (or: links) $E=E(G) \subset V \times V$. A sequence $\gamma=\left(v_{0}, v_{1}, \ldots, v_{n}\right)$ is called a path in $G$ of length $n$, if $\left(v_{i}, v_{i+1}\right) \in E$ for $0 \leq i \leq n-1$. Each edge $\left(v_{i}, v_{i+1}\right)$ is then said to belong to $\gamma$, and $\gamma$ is said to pass through $\left(v_{i}, v_{i+1}\right)$. If $v_{n}=v_{0}$, then $\gamma$ is said to be a cycle or a closed orbit of length $n$. If $v_{i} \neq v_{j}, \forall i \neq j$, then the cycle is said to be simple, irreducible, or fundamental. ${ }^{3}$ If $\gamma_{1}=\left(v_{0}, v_{1}\right.$, $\left.\ldots, v_{n}\right)$ and $\gamma 2=\left(w_{0}, w_{1}, \ldots, w_{m}\right)$ are two paths such that $v_{n}=w_{0}$, then the sequence $\gamma_{1} \gamma_{2}=\left(v_{0}, v_{1}, \ldots, v_{n}, w_{0}, w_{1}, \ldots, w_{m}\right)$ is again a path, of length $n+m+1$, called the product of $\gamma_{1}$ and $\gamma_{2}$. If $\gamma_{1}$ and $\gamma_{2}$ are cycles and $v_{i}=w_{j}$ for some $i, j$, then $\gamma_{1} \gamma_{2}$ is again a cycle, but no longer irreducible. Obviously, every cycle can be uniquely represented as a product of simple cycles. Vertices at which a sequence bifurcates, i.e., where $v_{i}$ offers two or more successors $v_{i+1}$, are called branching points and the cycles are called adjacent.

In the sequence space $X=V^{\mathbb{Z}}$ we can consider the subset of sequences $x=\left(x_{i}, i \in \mathbb{Z}\right)$ such that $\left(x_{i}, x_{i+1}\right) \in E(G)$, $\forall i \in \mathbb{Z}$. The shift or dynamical map $S$ on $X$ is defined as the mapping that transfers each sequence $v=\left(x_{i}, i \in \mathbb{Z}\right)$ into the sequence $w=\left(x_{i+1}, i \in \mathbb{Z}\right)$. The set $X(G)$ is obviously $S$ invariant. The pair $(X(G), S)$ is called the symbolic chain corresponding to the graph $G$. We can identify the symbolic chain with the invariant set and with the graph. By definition, the invariant set $X(G)$ consists of sequences in which each pair of neighboring symbols is admissible, that is, an edge of $G$. $V$ can be equipped with the discrete topology and $X$ with the direct product topology. For the latter, the finite-dimensional cylinders $\left\{x \in X: x_{i}=v_{n}, i=1, \ldots, k\right.$ form a base. $X(G)$ is closed in this topology and the shift $S$ is a homeomorphism. If the interpretation of $S$ is that the time evolution leads to a random journey on the graph structure, we obtain a "Markov random graph."
Upon enumeration, a graph $G$ with a finite number of vertices can be represented in the form of a transition matrix $\tilde{\mathbb{M}}$, from which all quantities of interest can be obtained. Any observable quantity that is associated with a symbolic sequence obtained from labeling the vertices can be transported only along admissible paths. ${ }^{8-10} \mathrm{~A}$ graph is called strongly connected if any node can be reached, after a number of steps, from any other node. Often, in applications, we deal with graphs that implement a drift (in D's courtship: the drift toward copulation). Starting anywhere on the graph, one is eventually being guided toward a sink (a node with only ingoing edges) from where there is no escape.

\section{MEASURES ON GRAPHS}

If the path itself is the only aspect of interest, we speak of the topological viewpoint, and the matrix $\tilde{\mathbb{M}}$ is the graphs adjacency matrix. If, however, we want or need to keep track of quantities that are multiplicative along the graph, but may split at branching points, such as probabilities or stabilities, we speak of a metric characterization. In the thermodynamic formalism, the inverse temperature $\beta$ provides a one-parametric family of measures that scans through all possible metric measures. ${ }^{8,11,12}$ This formalism allows for an in-depth statistical analysis of the dynamical system in the following sense. For systems without a drift, the underlying graph is generally strongly connected and has no sinks. To finite memory processes, finite non-negative matrices are associated, to which the Frobenius-Perron matrix theorem applies. The general theory states that the Frobenius-Perron matrix for ergodic, noncyclic processes is irreducible, the associated largest eigenvalue, $\lambda_{\max }$, analytically depends on its parameters (here: $\beta$ ), and phase transitions are prohibited. Phase transitions only occur for nonergodic finite systems, for ergodic cyclic systems, for infinite, or for infinite memory processes.

The presence of a drift triggers such a case. The probably simplest example is a two-symbol system $\{a, b\}$ with the only allowed transitions $\{a \rightarrow a, a \rightarrow b, b \rightarrow b\}$. This system is weakly connected and the associated transition matrix is substochastic and reducible. The associated transition matrix has two eigenvalues $\mu_{1}$ and $\mu_{2}$. The thermodynamic formalism ${ }^{8,11,12}$ systematically scans a family of measures by mimicking a "temperature" dependence of the matrix entries, by raising them to a power of $\beta$ (the "inverse temperature"). This also raises the matrix's eigenvalues to powers of $\beta$. The system behavior is characterized by the largest eigenvalue. As a function of $\beta$, the two eigenvalues have to cross., and we observe a first order phase transition, induced by the nonergodicity of the system. Unfortunately, for very complex systems, we are computationally limited with these techniques and they also do not lead to a straightforward description of a complex graph in terms of seizable simpler units.

\section{PERIODIC ORBIT APPROACH}

The usage of IUPO makes these complex systems finally amenable to an in-depth statistical physics analysis in the following way. The eigenvalues of the transition matrix 
$\tilde{\mathbb{M}}$ are determined from the characteristic equation involving a Fredholm determinant

$$
0=\operatorname{det}(1-z \widetilde{\mathbb{M}})=: \frac{1}{\zeta(z)},
$$

where $\zeta(z)$ is the dynamical zeta-function. ${ }^{11} \operatorname{det}(1-\mathbb{M})$ (where now $z$ is absorbed in the matrix $\mathbb{M}$ ) can be written as the sum of all possible partitions of the graph into products of nonintersecting loops, with each loop carrying a minus sign. ${ }^{15}$ For the graph displayed in Fig. 2, we obtain ${ }^{3}$

$$
\begin{aligned}
\frac{1}{\zeta}=\operatorname{det} & 1 \\
& -\mathbb{M})=1-m_{o_{1}} \\
& -m_{o_{2}} \\
& -m_{o_{3}} \\
& -m_{o_{4}} \\
& +m_{o_{1}} m_{o_{3}} \\
& +m_{o_{2}} m_{o_{3}},
\end{aligned}
$$

where the matrix entries $m$ capture the nature of the measure observed (as for the most common cases: topological properties, the transition probabilities, stability properties).

Note that the contributions are provided by the fundamental cycles plus all products among nonintersecting fundamental cycles. ${ }^{3,15}$ This is the reason why the cycle product $m_{O_{2}} m_{O_{3}}$ appears in the sum of Eq. (2), but not $m_{O_{1}} m_{o_{2}}$. A few cycles out of many thus contain the essential information. As is well-known, the trace $s p$ of $\mathbb{M}^{n}$ is the sum of all closed cycles of length $n$. If the Markov random graph is generated from a (chaotic) dynamical map $f$ with a Markov partition, we have the relation

$$
s p\left(M^{n}\right)=\int \delta\left(x-f^{n}(x)\right) d x=\sum_{x: f^{n}(x)=x} \frac{1}{|1-\Lambda(x)|},
$$

where $\Lambda(x)=(\mathrm{d} / d x) f^{n}(x)$ is the derivative of the dynamical map along a cycle of length $n$. If the map $f$ coincides with the shift map $S$, this leads to $\Lambda(x)=0, \forall x$. Equation (3) shows that in this case our description results in the trivial, cycle-counting, measure, which is at the base of the topological entropy. ${ }^{8}$ For the example at hand, we obtain

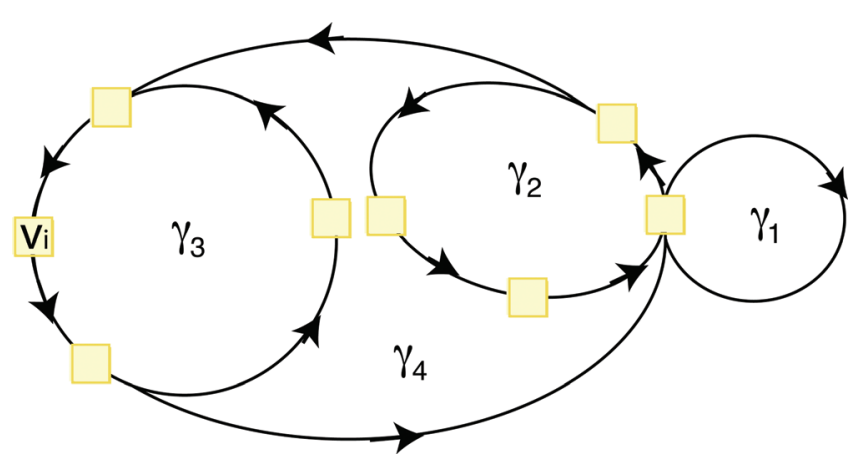

FIG. 2. (Color online) Example of a directed graph and its decomposition into four fundamental cycles (or IUPO).

$$
0=1-z-2 z^{4}+z^{8}
$$

which yields a topological entropy of $h_{\text {top }}=-\log (z$ $=0.658779 \ldots)=0.417367 \ldots$ For a complete binary dynamics (e.g., from the Bernoulli shift map), we would similarly obtain

$$
\frac{1}{\zeta}=1-2 z
$$

This concludes our demonstration that from the IUPO, the characteristic properties of the complex network can efficiently be extracted, whenever they make sense. Note that in the dynamical system approach, there is a strong meaning in a single time step, as a sort of a physical clock that is transported along the path. If there is no intrinsic meaning to be attached to this "natural" time-step, a distinction between shorter and longer cycles is not necessary and fundamental cycles could be concatenated into one single symbol each.

\section{NOISY GRAPHS}

If strong noise is present, precision-dependent characteristics lose their meaning. Nonadjacency of two fundamental cycles is one of those. In this case, the distinction between intersecting and nonintersecting fundamental cycles can be abandoned (this view is moreover supported by the large number of cycles present, the higher order in $z$ of the cycle products, and the absence of a natural time step as outlined above). Written correspondingly to Eq. (2), the system is now represented by a low-order approximated expansion of the Fredholm determinant

$$
\frac{1}{\zeta}=\operatorname{det}(1-\mathbb{M})=1-\sum_{i=1}^{m} o_{i}
$$

where $o_{i}$ are the fundamental cycles found in the system. A graphical sketch of this situation is provided in Fig. 3. Our application will demonstrate that, despite this simplification, this approach is able to work out an improved degree of insight into the system behavior.

\section{GRAPH SIMILARITY}

If several such graphs are to be compared, this comparison therefore takes place on the basis of lists of cycles. To facilitate this comparison, we write the lists as vectors, so that a system $M_{i}$ is represented as

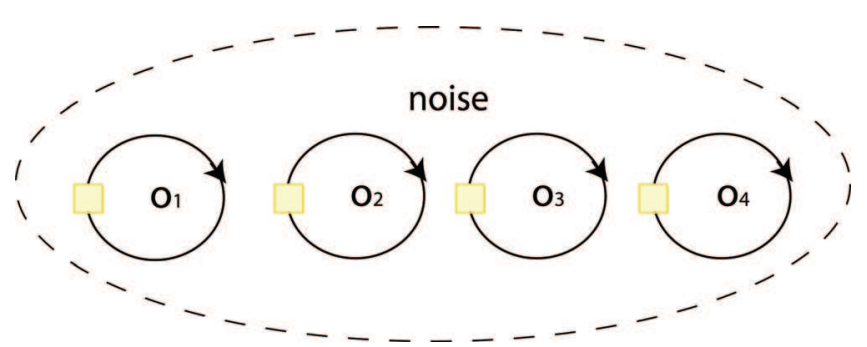

FIG. 3. (Color online) Simplified model for noisy systems (for the original system see Fig. 2): The fundamental cycles are concatenated to single elements, all connected by noise, so that no additional products emerge. 


$$
M_{i}: \vec{m}_{i}=\left(\gamma_{p(i, 1)}, \gamma_{p(i, 2)}, \ldots, \gamma_{p\left(i, n_{i}\right)}\right)^{T},
$$

where the indices $k=1 \ldots n_{i}$ labels the different closed orbits (or products $p(i, k)$ of closed orbits if this makes sense) of the $i$ th system. In the following, we restrict ourselves for simplicity to the comparison of systems that are based on a common underlying set of symbols, although our approach is more general. Different graphs will generally give rise to different sets of fundamental cycles. Hence, they will lead to generally incomparable vectors $\vec{m}_{i} \neq \vec{m}_{j}, i \neq j$. Therefore, first a space needs to be defined in which a comparison among these vectors will make sense. A suitable space is obtained from the union $U$ of all fundamental cycles (and suitable products, if this makes sense) $\gamma_{p(i, k)}$, collected from all graphs entering the comparison

$$
U=\cup_{i=1}^{N} \cup_{k=1}^{N_{i}} \gamma_{p(i, k)},
$$

where $N$ denotes the numbers of systems and $N_{i}$ the number of contributions from the system indexed by $i$. With each element of $\gamma_{1} \in U$, a vector $\vec{v}_{l}$ of length $\kappa=$ card $(U)$ can be associated by the mapping

$$
\gamma_{l} \leftrightarrow \vec{v}_{l}=(0, \ldots, 0,1,0, \ldots, 0)^{T}
$$

where the only nonzero entry is the component indexed by $l$. The set of these vectors can be used as a basis for a vectorspace $\mathbb{V}$, in which $M_{i}$ is expressed by a vector

$$
\overrightarrow{b_{i}}=\left(\tilde{v}_{1}, \tilde{v}_{2}, \ldots, \tilde{v}_{\kappa}\right)^{T}
$$

where $\tilde{v}_{l}=\gamma_{p(i, k)}$ if $\exists k: \quad \gamma_{p(i, k)}=\gamma_{l}$ and $\tilde{v}_{l}=0$ otherwise. The vector nature of $\vec{b}$ suggests that the inner product $\left\langle\overrightarrow{b_{j}}, \overrightarrow{b_{i}}\right\rangle$ provides a simple way for assessing the "similarity" between two graphs, where it should be noted that this notion of similarity is only an approximate one that does not include transitivity. In the following analysis of the Drosophila courtship, as our application, we consider only the topological aspect. The information of how many times a node is visited, e.g., is ignored. More details for why this is appropriate in the context of this application are provided in Ref. 6 .

\section{DROSOPHILA COURTSHIP}

There is wide agreement that a major percentage of every day human information exchange is nonverbal ("body language," gesture, sound, smell, appearance,...). Despite the obvious interest, the question of what properties body language shares with spoken language has attracted little attention, which is probably due to the lack of appropriate methods. A characteristic element of spoken languages is grammar. Why grammatical rules are needed for communication is not evident at all. Linguist Noam Chomsky made the argument that the human brain contains a genetically determined limited set of rules for organizing language, implying that all human languages have a common structural basis, a set of rules known as the Universal Grammar. ${ }^{13}$ Another suggestion - emphasized by physics - is that grammar emerges from the natural interdependence between things that we perceive, which only allows for a small subset of all theoretically possible relations to be realized. Finally, by grammar, redundancy can be introduced, which allows for an efficient check of correctness for transmitted information.

A particular case of body language is $P C$. Before mating, most animals engage in extensive $P C$ activities, the costs of which are generally high for the individual. One hypothesis may therefore be that $P C$ relates to aims central for the survival of the species. An obvious suggestion is that $P C^{\prime}$ 's role could be the exchange of information about the genetic suitability of a prospective mate.

In order to obtain $D$ 's courtship graph, we recorded and investigated high-speed movies of DPC between two individual flies of generally different genetical and developmental conformations. The recorded courtship behaviors were analyzed and each one separated into 37 fundamental (on neuronal time scales irreducible and mutually independent) acts. ${ }^{6}$ These are the nodes of the courtship graph that emerges from connecting sequential acts. We now look at $D P C$ as a dynamical system, where the $P C$ fundamental acts correspond to the symbols used in the symbolic dynamics. In our experiments, we compared the body language of animals of different genetical conformations in different partner contexts. ${ }^{14}$ From the courtship video data, we labeled each beginning of a fundamental act of a protagonist by the corresponding symbol number. In this way, we obtained from each courtship experiment two files (for the two protagonists) of sequences of numbers $1 . .37$ (see the Appendix for details). In Fig. 1, we display the undirected courtship graph composed from all individuals observed. This graph represents the behavioral space of $D P C$. Special groups (e.g., male or females) have accession to certain parts thereof, see Fig. 4. Instead of a random choice in compliance with the probability transition matrix, transitions reflect the interaction taken by two courtship protagonists. From an ensemble view, the average over many such observations could again be captured by a probability transition matrix.

For the IUPO analysis, we started with the first symbol of the file and scanned through the file, until a first repetition of the symbol is found. Then, this is repeated for the following symbols. The procedure is performed such that

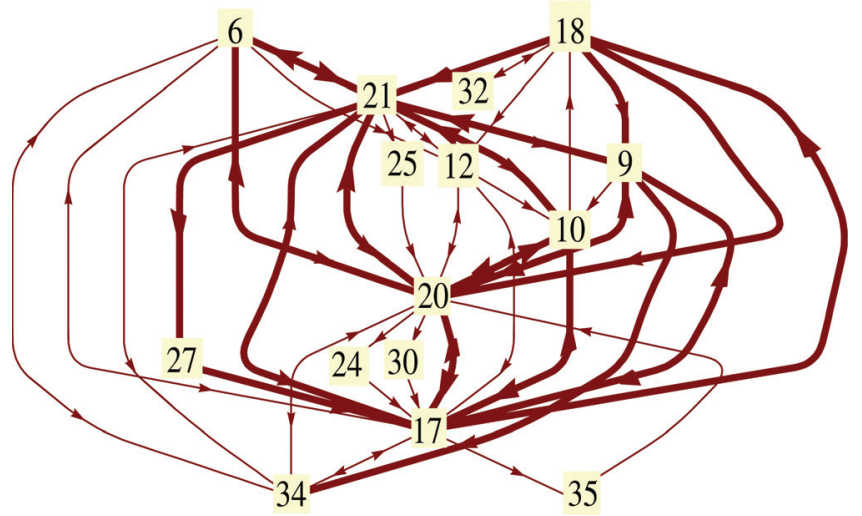

FIG. 4. (Color online) Directed $P C$ subgraph of mature female as the protagonist, with normal males (full graph) and with fruitless males (bold lines, a subgraph of the first) as partners. 

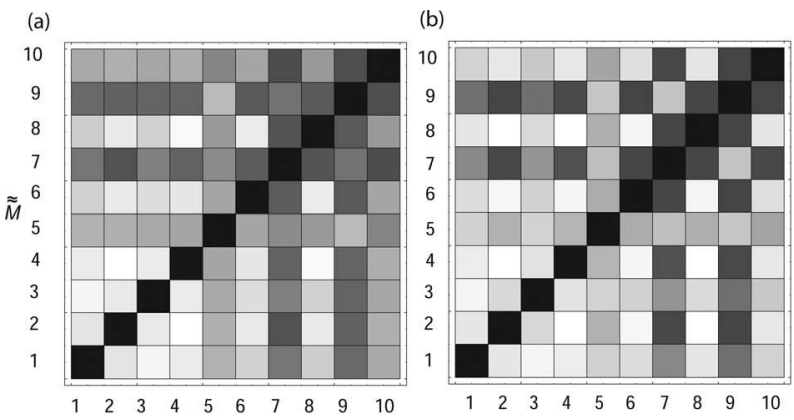

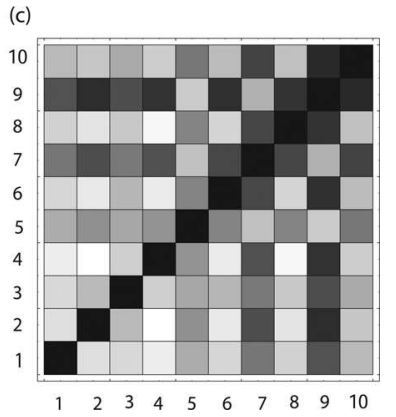

FIG. 5. Class (Ref. 14) similarities, density plots over $[0$, maxcorr $]=[0,1]$. Lighter shading indicates higher similarities, diagonal elements were set to 0 . (a) Original data with closed orbit analysis, (b) surrogate data with closed orbit analysis, (c) symbol probability vectors. The original data with closed orbit analysis works out best in the class dissimilarities among "normal" classes and the indifferent behavior among the fruitless classes $1-4$. See Ref. 6 for more details. automatically only irreducible (indecomposable) orbits are collected. From the whole of the available experimental data, we obtained 181 irreducible closed orbits. Each protagonist was thus described by 181-dimensional vector, containing as an entry 1 if the corresponding orbit was present in the data, and 0 otherwise. For obtaining a characterization of each experimental group, the vectors were pooled. The values shown in Figs. 5(a) and 5(b) were obtained by taking the scalar products among the normalized pooled vectors. Considering as a basis instead of closed orbits solely symbol probabilities leads to Fig. 5(c). More details, in particular referring to the pooling process, can be found in Ref. 6 .

From the analysis, classes of behavior emerge that reliably reflect the genetical conformations of the protagonist and of the partner (for genetically healthy animals). Moreover, the behavioral classes follow intricate grammars that cannot be the result of chance. The grammatical richness we measure by the abundance of repeating structures (i.e., periodic orbits), in comparison to those appearing in surrogate data sets, i.e., randomly generated networks based on the same probabilities of symbols. We find an overwhelming richness of repeating structures that outnumber by far what is observed in the surrogate data. We find grammatical structures even, but to a much lesser extent, in the behavior of genetically aberrant animals.

\section{DETAILED RESULTS}

The extent by which closed orbits (or the grammar they embody) would be beneficial for the identification of the experimental classes of $D$ was of particular interest. After characterizing each file by means of its behavioral vector $\vec{b}$, we calculated the scalar product between the different vectors, and, in order to compare class behavior, we also considered class behavior by averaging over the protagonists of each class (this pooling can be justified ${ }^{6}$ ). We found that despite an extremely large variability of individual behaviors, to the vast majority of the individuals, the classes are distinguishable. $^{6}$ This supports the hypothesis that $P C$ conveys mating suitability, including individual profiles. On the class level, the areas of greatest dissimilar behavior (dark bands and dark subdiagonal parts) indicate a maximally spread communication code between normal males and females, which we believe cannot be accidental.

To investigate the role of periodic orbits for the distinction among classes or individuals, we characterized in a
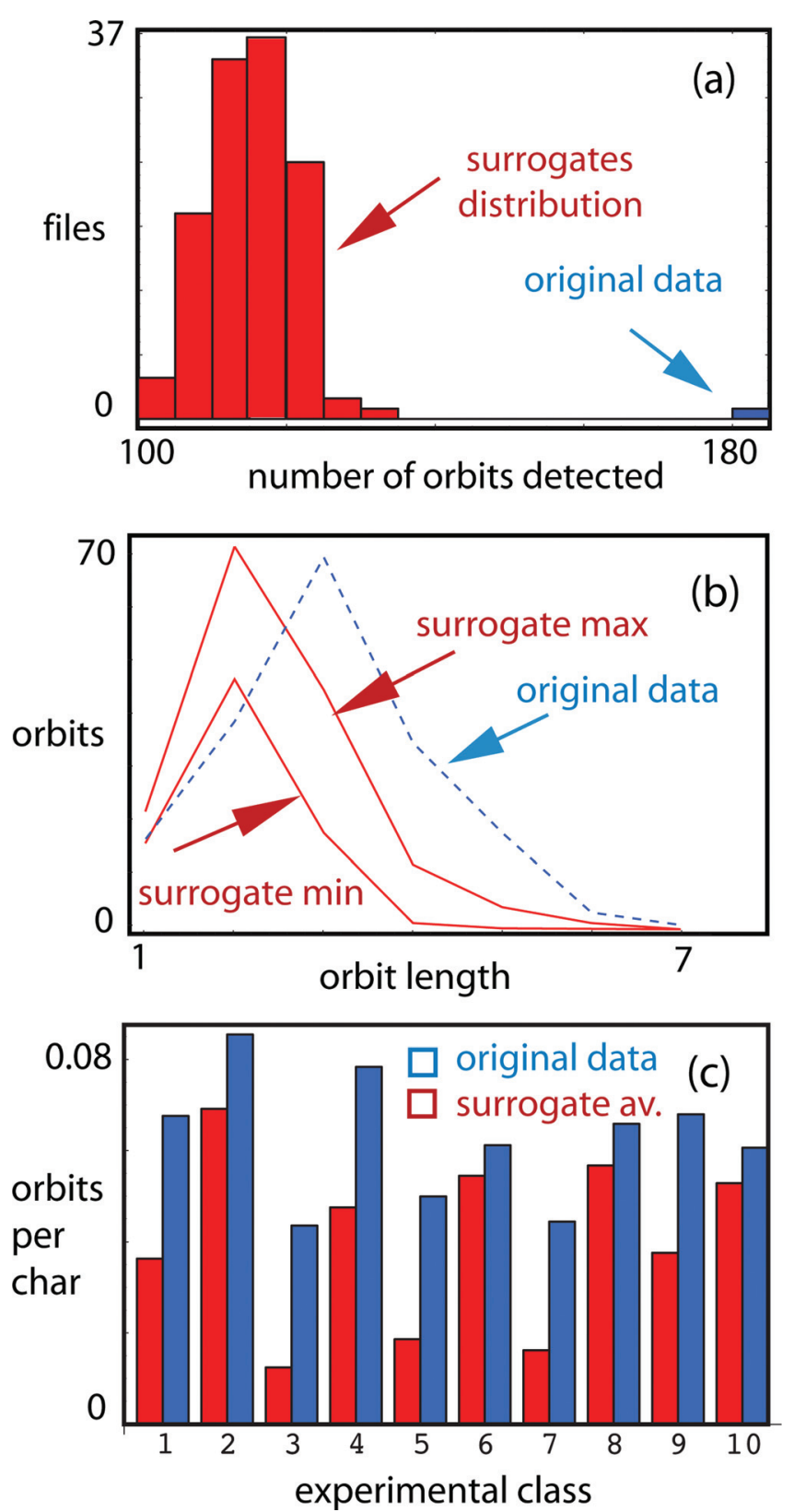

FIG. 6. (Color) Closed orbits found in the original and in 120 surrogate files. (a) Histogram of the number of (different) closed orbits found in the surrogates. (b) Maximal/minimal number of orbits of a given length found in the surrogates. (c) Number of closed orbits per character for the different experimental classes (Ref. 14) (surrogate average). Surrogates (red) contain substantially fewer orbits than the original files (blue). Roughly one third of the orbits found in a surrogate file are not present in the original data. 
second model, each protagonist by a behavioral vector the entries of which were the natural symbol probabilities, i.e., had the form $\overrightarrow{b_{i}}=\left(w_{1}, w_{2}, \ldots, w_{37}\right)$, where $w_{i}, i=1 \ldots 37$ are the symbol probabilities in the class considered. This data model is based on the trivial grammar (no grammatical rules). Remarkably, the characteristic stripes and peaks emerge much at the same places even if behavior is described by the probability of symbols only [Fig. 5(a) versus Fig. 5(c)]. As this characterization does not take into account any aspects of grammar, for expressing the similarities/dissimilarities among the classes the closed orbits cannot be crucial. Neither did we find any salient differences when we compared our experimental data with a periodic orbit analysis of surrogate models based on the given symbol probabilities [Fig. 6(a) versus Fig. 6(b)]. The surrogate method will typically provide the most unspecific model compatible with the given distribution, i.e., the one with the most general, i.e. unrestricted, grammar.

If the closed orbits would have a beneficial role for efficient communication at all, we would, however, expect to find a largely increased number of irreducible closed orbits in the original data, indicative of a nontrivial grammar. We therefore compared how many closed irreducible orbits are present in the original data versus the surrogate case (in terms of numbers of distinct orbits, not of frequencies). The results of Fig. 6 clearly hallmark the presence of a highly developed grammar underlying $P C$. Detailed investigations yield that a large portion of the orbits found in the original data are a priori unlikely ones. These findings justify the statement that a specific, nonaccidental, structure, which can be interpreted as a grammar, underlies $D P C$.

\section{CONCLUSION}

Our general construction by which individual networks are embedded into an all-embracing network, allows for the comparison of networks that share a variable number of common elements. By taking into account the specific ordering in which the elements appear, our characterization reaches beyond the pure presence of node elements. This is necessary in order to bind isolated actions toward a characterization of behavior.

The described mesoscopic analysis is general enough to be applicable to a wide variety of comparative studies of branching processes on complex graphs that critically depend upon a sufficiently detailed quantitative analysis. In Neuroscience, e.g., the method may be used to pin down the neural origins of behavior, by relating the behavioral elements or motifs to the corresponding processes in the working brain.

The application to $D P C$ provides a proof of concept and highlights the strength of the approach. It reveals a highly variable and specific $D P C$ language, providing the individual with a large bandwidth communication platform, a situation that has a close resemblance to chaotic communication setups. ${ }^{16-24}$ Male $D$, in particular, widely uses this expression space, which indicates that during courtship, more than mere group membership is conveyed. Transferred information might even include behaviorally encoded details on the sender's genetic configuration, as the base for situation-dependent decisions such as the "better mate versus no mate" trade-off.
It is also suggestive that the redundancy implemented via the orbit structure, enhances reading reliability, as is indicated in Fig. 5. To uncover such fine-structures, a refined, mesoscopic, network analysis was indispensable.

\section{APPENDIX: METHODS}

\section{Fly stocks}

The Drosophila melanogaster flies used in this study are of the wild-type Canton S (CS) strain and mutants carrying an allele of the fruitless mutation $\left(f r u^{1}\right) .{ }^{25}$ Raised on a cornmeal/agar/molasses/yeast medium at $25{ }^{\circ} \mathrm{C}$ on a $12: 12 \mathrm{~L}: \mathrm{D}$ cycle, the flies were sexed and the naïve males and virgin females were isolated within $2 \mathrm{~h}$ after eclosion under cold anesthesia $\left(4^{\circ} \mathrm{C}\right)$. Until recording, the males were kept individually in test tubes, where mature male and female flies used for the recording were 4-5 days old. The mutant males used in the assays were homozygous for $f r u^{1}$.

\section{Data acquisition}

A pair of flies was transferred, by aspiration, into a cylindrical mating chamber of dimensions $0.8 \mathrm{~cm}$ diameter and $0.5 \mathrm{~cm}$ height. Environmental conditions were fixed at $25{ }^{\circ} \mathrm{C}$ and $75 \%$ humidity. A 5-min episode (or until copulation in the case of the mature virgins) was recorded with a Sony Hi8 video/audio camera, using 29.97 frames per second. The videotape recordings were converted from Hi8 to digital video, and then compressed and converted into MPEG 1 format, using CLEANER 6 software (Discreet, New York, NY). The files were loaded onto the THEME coder (Patternvision Inc., Iceland) and analyzed frame by frame. The beginning and ending of every act was systematically registered, for both protagonists (resulting in two files, one for each protagonist). The data were analyzed frame by frame (30 frames per second) using an interactive multimedia program. The beginning and ending of every behavior was systematically registered for the male and female of each pair to a $1 / 30$ of a second resolution. At this time resolution, all neuronally relevant visual acts can be resolved.

\section{Fundamental behavioral acts}

Our criterion was that any continuous action with a beginning and ending that was clearly observable at the temporal resolution of 30 frames per second would fit our definition of behavior element. The high spatial and temporal video resolution enabled us to observe very fast action elements. Not all of the described elements were found in every data set. Behavioral acts for which, because they are independent, further dissection makes no sense, are termed fundamental. The full list of fundamental acts is displayed in Table I; their descriptions are as follows.

\section{a. Female behavior elements}

Abdotwist: A twisting of the abdomen sideways and downward.

Kicking: Applying tarsi forcefully against partner.

Ovipositor extrusion: Telescoping extension of ovipositor. 
TABLE I. Encoding scheme: 37 fundamental acts found in the time series are encoded into numbers, some of which are sex-specific. For some illustrations, see Ref. 26.

\begin{tabular}{|c|c|c|}
\hline \multicolumn{3}{|c|}{ Drosophila acts } \\
\hline 1 & Abdobend & Female \\
\hline 2 & Abdotwist & Female \\
\hline 3 & Attemptcop & Male \\
\hline 4 & Circling & Male \\
\hline 5 & Copulation & Male \\
\hline 6 & Decamp & Male/female \\
\hline 7 & Fencing & Male \\
\hline 8 & Following & Male \\
\hline 9 & Grooming forlegs & Male/female \\
\hline 10 & Grooming hindlegs & Male/female \\
\hline 11 & Headpos & Male/female \\
\hline 12 & Kick hindlegs & Female \\
\hline 13 & Licking & Male \\
\hline 14 & Orientation & Male \\
\hline 15 & Ovipext & Female \\
\hline 16 & Run & Male/female \\
\hline 17 & Standing & Male/female \\
\hline 18 & Still & Male/female \\
\hline 19 & Tapping & Male \\
\hline 20 & Walk left & Male/female \\
\hline 21 & Walk right & Male/female \\
\hline 22 & Wingext left & Male \\
\hline 23 & Wingext right & Male \\
\hline 24 & Wingflicks left & Male/female \\
\hline 25 & Wingflicks right & Male/female \\
\hline 26 & Wingflutter & Female \\
\hline 27 & Wingspread & Male/female \\
\hline 28 & Wingwave & Male \\
\hline 29 & Wingflicks unspec. & Male/female \\
\hline 30 & Grooming midlegs & Male/female \\
\hline 31 & Tapping forelegs & Male \\
\hline 32 & Kick midlegs & Female \\
\hline 33 & Walk unspec. & Male/female \\
\hline 34 & Kick unspec. & Male/female \\
\hline 35 & Wingflap & Male/female \\
\hline 36 & Run right & Male/female \\
\hline 37 & Run left & Male/female \\
\hline
\end{tabular}

Wiggle: Wagging of abdomen and wings in opposite directions.

Wingflutter: Left and right flicks in succession.

b. Male behavior elements

Orientation: Fly is facing the partner, and may involve little motion such as turning to keep facing the partner, leaning over or no motion.

Following: Locomotion in directed pursuit of a partner.

Tapping: Touching partner with tarsi of prothoracic forelegs.

Fencing: Face-to-face and engaged in sparring with prothoracic forelegs.

Wing extension: Wing stretched out away (i.e., perpendicular to the head abdomen axis) from the body biased to the left or right.

Circling: A sideways skid along a semicircular path around a partner.
Licking: proboscis contact with partner.

Abdominal bending (Abdobend): Abdominal curling under thorax toward the head direction.

Attempted copulation: Abdominal curling directed toward a partner.

Copulation: Sustained genital connection between male and female.

\section{c. Common behavior elements}

Abdominal vibration: Vertical up and down movement of the abdomen.

Decamp: An evasive jump away from partner, usually involving a somersault.

Grooming: Rubbing of tarsi together, forelegs, midlegs, or hindlegs.

Standing: Absence of locomotion with no indication of activity directed toward the partner.

Still: No detectable movement of any body parts during standing.

Walking (walk): Locomotion that is not directed toward courting the partner.

Wing flicks: Brief spasmic movement of wings to and from the antero-posterior axis of the body in rapid succession.

Wing scissoring: Both wings moved away from and back to the body in a rapid scissor-like manner.

Wing spread: Both wings are stretched out away from the body to give a wide $\mathrm{V}$-shape forming an angle of at least $90^{\circ}$ that is bisected by the anterior to posterior axis of the fly. This set of acts extends earlier descriptions. $^{27-29}$

\section{Behavioral experiments and classes}

In our behavioral experiments, normal females in the immature, mature, and mated states were paired with normal males in an observation chamber (yielding a set of six distinct protagonist roles). In extension of these experiments, also fruitless $^{30}$ mutant males were paired with either mature females or mature normal males (resulting in four more protagonist roles). The emergent courtship behavior has tactile, gustatory, olfactory, acoustic, and visual sensory dimensions, ${ }^{31,32}$ with the olfactory and the visual components being most salient, ${ }^{26,29,33,35}$ where the latter is easy to access, which is why our analysis focused on that component. Although our analysis is based on time series of one protagonist at a time, we will obtain also considerable insight into the interplay between partners. The experimental classes with the indices used for their abbreviation are shown in Table II.

In order to eliminate disambiguation in the definition of the behavioral states, we used visual recordings of the almost neuronal temporal resolution of 30 frames per second. The starting points of every act were detected using frame-byframe inspection, and indexed using the symbols of Table I. As a further increase of this temporal resolution provides no further information, we refer to the coded acts as fundamental. For each possible experimental constellation, five trials of the experiment were performed, using different 
TABLE II. Correspondence between indices and $D$ behavioral vectors (see text).

$1 \hat{=}$ fruitless males in the presence of mature females

$2 \hat{=}$ mature females in the presence of fruitless males

$3 \hat{=}$ fruitless males in the presence of normal males

$4 \hat{=}$ normal males in the presence of fruitless males

$5 \hat{=}$ normal males in the presence of mated females

$6 \hat{=}$ mated females in the presence of normal males

$7 \hat{=}$ normal males in the presence of mature females

$8 \hat{=}$ mature females in the presence of normal males

$9 \hat{=}$ normal males in the presence of immature females

$10 \hat{=}$ immature females in the presence of normal males

individuals. The individuals were not previously screened for whether they would court or not. In this way, out of 50 protagonists we lost two, by a fruitless/mature female experiment that produced no usable results.

\section{Surrogate data analysis}

For the surrogate data, we shuffled the data within individual files by means of permutations. Note that in this way, an increased tendency for periodic orbits occurs (since successive repetitions of some of the symbols are very unlikely) that does not show up in the original files. The displayed results were not corrected for this effect. A corresponding correction, however, preserves the reported findings.

${ }^{1}$ D. Auerbach, P. Cvitanovic, J.-P. Eckmann, and G. Gunaratne, Phys. Rev. Lett. 23, 2387 (1987).

${ }^{2}$ P. Cvitanovic, Physica D 51, 138 (1991).

${ }^{3}$ P. Cvitanovic, Physica D 83, 109 (1995).

${ }^{4}$ P. So, E. Ott, S. J. Schiff, D. T. Kaplan, T. Sauer, and C. Grebogi, Phys. Rev. Lett. 76, 4705 (1996).

${ }^{5}$ V. S. Afraimovich and L. A. Bunimovich, Nonlinearity 20, 1761 (2007).

${ }^{6}$ R. Stoop and B. I. Arthur, Chaos 18, 023123 (2008).

${ }^{7}$ R. Stoop and B. I. Arthur, "An expressive body language underlies Drosophila courtship behavior," in Understanding Complex Systems: Complex Dynamics in Physiological Systems: From Heart to Brain, edited by S. K. Dana, P. K. Roy, and J. Kurths (Springer, New York, 2009), pp. 215-227.
${ }^{8}$ J. Peinke, J. Parisi, O. E. Roessler, and R. Stoop, Encounter with Chaos (Springer, Berlin, 1992).

${ }^{9}$ R. Stoop, N. Stoop, and L. A. Bunimovich, J. Stat. Phys. 114, 1127 (2004).

${ }^{10}$ R. Stoop and N. Stoop, Chaos 14, 675 (2004).

${ }^{11}$ D. Ruelle, Dynamical Zeta Functions for Piecewise Monotone Maps of the Interval (AMS, Providence, Rhode Island, 1994).

${ }^{12} \mathrm{~T}$. Tél, Directions in Chaos, edited by Bai-lin Hao (World Scientific, Singapore, 1991), Vol. 3, pp. 149-221.

${ }^{13}$ G. F. Marcus, A. Vouloumanos, and I. A. Sag, Nat. Neurosci. 6, 651 (2003).

${ }^{14}$ The following experimental classes were considered: fruitless males ("fm.") in the presence of ("itp.") mature females ("matuf."): 1; matuf. itp. fm.: 2; fm. itp. normal males ( "nm."): 3; nm. itp. fm.: 4; nm. itp. mated females ( "matedf."): 5; matedf itp. nm.: 6; nm. itp. matuf.: 7; matuf. itp. nm.: 8; nm. itp. immature females ("immf."): 9; immf. itp. nm.: 10.

${ }^{15}$ D. M. Cvetcovic, M. Doob, and H. Sachs, Spectra of Graphs (J. A. Barth Verlag, Heidelberg-Leipzig, 1995).

${ }^{16}$ S. Hayes, C. Grebogi, and E. Ott, Phys. Rev. Lett. 70, 3031 (1993).

${ }^{17}$ K. Pyragas and A. Tamasevicius, Phys. Lett. A 180, 99 (1993).

${ }^{18}$ H. D. I. Abarbanel and P. S. Linsay, IEEE Trans. Circuits Syst. 40, 643 (1993).

${ }^{19}$ A. Loskutov, V. M. Tereshko, and K. A. Vasiliev, Int. J. Bifurcation Chaos 6, 725 (1996).

${ }^{20}$ Yu. V. Andreev, A. S. Dmitriev, and S. O. Starkov, IEEE Trans. Circuits Syst. 44, 21 (1997).

${ }^{21}$ L. Kocarev and G. Jakimovski, IEEE Trans. Circuits Syst. 48, 163 (2001).

${ }^{22}$ B. Fraser, P. Yu, and T. Lookman, Phys. Rev. E 66, 017202 (2002).

${ }^{23}$ A. Loskutov, S. D. Rybalko, and A. A. Churaev, Tech. Phys. Lett. 30, 843 (2004).

${ }^{24}$ A. Loskutov and S. D. Rybalko, J. Commun. Technol. Electron. 50, 1358 (2005).

${ }^{25}$ J. C. Hall, Science 264, 1702 (1994).

${ }^{26}$ J. Billeter, E. Rideout, A. Dornan, and S. Goodwin, Curr. Biol. 16, R766 (2006).

${ }^{27}$ R. J. Greenspan and J. F. Ferveur, Annu. Rev. Genet. 34, 205 (2000).

${ }^{28}$ C. Lasbleiz, J. F. Ferveur, and C. Everaerts, Anim. Behav. 72, 1001 (2006).

${ }^{29}$ T. A. Markow, Proc. Natl. Acad. Sci. U.S.A. 84, 6200 (1987).

${ }^{30}$ D. A. Gailey and J. C. Hall, Genetics 121, 773 (1989).

${ }^{31}$ H. T. Spieth, Annu. Rev. Entomol. 19, 385 (1974).

${ }^{32}$ L. Giarratani and L. B. Vosshall, Neuron 39, 881 (2003).

${ }^{33}$ H.-G. Lee, Y.-C. Kim, J. S. Dunning, and K.-A. Han, PLoS One 3(1), e1391 (2008).

${ }^{34}$ H. V. Hirsch and L. Tompkins, J. Exp. Biol. 195, 1 (1994).

${ }^{35}$ I. Shmulevich, E. R. Dougherty, S. Kim, and W. Zhang, Bioinformatics 18, 261 (2002). 